\title{
WG7 Highlights - Future DIS Experiments
}

\author{
Néstor Armesto*计 \\ University of Santiago de Compostela, Departamento de Física de Partículas and IGFAE, Spain \\ E-mail: nestor.armesto@usc.es
}

\section{Armen Buniatyan}

Ruprecht-Karls University of Heidelberg, Physics Institute, Germany

E-mail: armen.buniatyan@desy.de

\section{F. Sabatié ${ }^{\S}$}

IRFU/Service de physique Nucléaire, CEA Saclay, F-91191 Gif-sur-Yvette, France

E-mail: franck.sabatiedcea.fr

\begin{abstract}
We summarise the contributions to Working Group 7 - Future DIS Experiments, presented during this conference. Contributions are divided into those related with the EIC projects, those related with the $\mathrm{LHeC}$ project, and all others.
\end{abstract}

XXI International Workshop on Deep-Inelastic Scattering and Related Subject -DIS2013,

22-26 April 2013

Marseille,France

\footnotetext{
* Speaker.

${ }^{\dagger}$ The work of NA is supported by European Research Council grant HotLHC ERC-201-StG-279579; by Ministerio de Ciencia e Innovación of Spain under projects FPA2009-06867-E and FPA2011-22776; by Xunta de Galicia (Consellería de Educación and Consellería de Innovación e Industria - Programa Incite); by the Spanish Consolider-Ingenio 2010 Programme CPAN and by FEDER.

¥Speaker.

${ }_{\text {Speaker. }}$
} 


\section{Status of the Electron-Ion Collider EIC}

The US Electron-Ion Collider project was extensively reviewed during this conference, with 6 contributions. Even though a lot of material covered could be found either in the Science Case document from the INT 2010 workshop [1] as well as the more recent EIC White Paper [2], the speakers also reported on some recent progress, especially on the technical developments towards such a collider. The EIC machine is aimed at achieving highly polarized ( $70 \%)$ electron and nucleon beams, ion beams from deuteron to the heaviest nuclei (uranium or lead), a variable center of mass energy from 20 to $100 \mathrm{GeV}$, upgradable to $150 \mathrm{GeV}$, a high collision luminosity of $10^{33-34} \mathrm{~cm}^{-2} \mathrm{~s}^{-1}$ and the possibility of having more than one interaction region.

A. Deshpande did a thorough overview of EIC, from the physics case to the description of the BNL and Jefferson Lab developments to build an electron-ion facility in two stages [3]. The EIC would help answering the following burning questions of QCD : How are the sea quarks, gluons, and their spins distributed in space and momentum inside the nucleon? Where does the saturation of gluon densities set in? How does the nuclear environment affect the distribution of quarks and gluons and their interactions in nuclei? A future (variable) high-energy and high-luminosity Electron Ion Collider (EIC) with possibility of colliding polarized electrons with polarized nucleons/lightnuclei, and a wide range in nuclear species is being considered by the US nuclear science community which promises to quantitatively address and resolve the above mentioned "profound and compelling questions" in QCD.

One of the essential aspects of the physics which can be achieved with an EIC concerns nuclei. Matthew Lamont reported on the insights in QCD which can be learned from e+A collision in the small-to-intermediate $x_{B}$ range [4]. Not only will nuclear PDFs be extracted, but the high statistics data generated will allow the study of the nucleus in fine detail. At high-x, the fragmentation of fast-moving partons in a nuclear environment will be investigated, something which has particular resonance to jet studies at RHIC and the LHC. At small- $x_{B}$, nucleon data have shown that gluons dominate at intermediate-to-small $x_{B}$. Indeed, it is believed that at some point, this growth is so large that it cannot grow any larger and it will saturate, giving rise to the saturation phenomena.

Elke-Caroline Aschenauer reported on the description of the BNL solution for the EIC called eRHIC, which would make use of the existing RHIC infrastructure [5]. eRHIC is a triple interaction point (IP) collider, with a dedicated eRHIC detector and the possibility of using the two existing - but upgraded - IP detectors PHENIX and STAR. The eRHIC machine was described, followed by an overview of the work related to interaction regions and dedicated detector designs as well as a short overview about the current research efforts for making technology choices towards such a new facility, including the especially important problem of the electron cooling of the proton beam.

The evolution of the PHENIX detector for EIC was presented by Klaus Dehmelt [6]. It is envisioned to fully use the sPHENIX detector at midrapidities $(|\eta|<1)$, followed by a sPHENIXForward upgrade with additional modifications specific to ePHENIX. The speaker showed the ePHENIX approach and goals as well as the detector requirements for day-1 physics at a stage1 eRHIC.

Yuhong Zhang review the corresponding Jefferson solution for the EIC after its $12 \mathrm{GeV}$ upgrade [7] : a polarized medium energy electron-ion collider (MEIC). Over the last three years, a conceptual design of MEIC has been successfully developed, and a comprehensive report summa- 
rizing the baseline and accelerator R\&D progress has been released recently. The MEIC design studies have now entered a new phase, focusing primarily on design optimization, and critical accelerator R\&D to prove the design concept. An overview for the MEIC design was shown with a focus on the recent progress of the accelerator design studies, in particular regarding the electron cooling again.

Pawel Nadel-Turonski followed with a complete description of the detectors required for the Jefferson Lab MEIC to achieve its full potential [8]. Indeed, one needs a full-acceptance detector able to detect all fragments, nuclear or partonic, including spectators, with high resolution, and to provide a wide coverage in $-t$ for recoil baryons from exclusive (diffractive) reactions at all beam energies. The author described how this is achieved at Jefferson Lab for both the central detector as well as the forward and very forward part, and how tightly this is integrated in the machine lattice.

As a summary, the US EIC community is growing strong and the EIC project is foreseen to be examined by NSAC in 2014-2015. In the meantime, a lot of developments both on the accelerator and detector sides are going on at BNL, Jefferson Lab, but also many places in US and even Europe, to get ready to build the future of nuclear physics and start taking data as early as 2025. In parallel, the physics program has nicely matured and covers all the critical questions required to finally understand the theory of strong interaction, QCD.

\section{Status of the LHeC}

The status of the Large Hadron-electron Collider (LHeC) and its most recent developments, were reviewed at the conference. All in all, 6 contributions on behalf of the Study Group [9, 10, 11, $12,13,14]$ and one more related with physics at the LHeC [15], were presented. They contained material previously released in the Conceptual Design Report [16] and contributed to the European Strategy Update [17] plus some more recent items [18].

Oliver Brüning [10] reviewed the accelerator project from the initial constraints to the very recent developments. Concerning the latter: the choice of optimal radiofrequency to be 801.58 MHz, the celebration of a workshop in 2013 at Daresbury (UK) on the LHeC Energy Recovery Linac (ERL) Test Facility, and the reconsideration of the luminosity expectation. It was concluded that, with final proton parameters for the High Luminosity (HL) phase of the LHC, luminosities around $10^{34} \mathrm{~cm}^{-2} \mathrm{~s}^{-1}$ can be achieved, a factor ten larger than the initial design luminosity. Finally, the possibilities for installation during and start after Long Shutdown 3 in the mid-2020's, were revisited. The synergies with HL-LHC and TLEP for the RF system were pointed out.

David South [14] reported on the detector studies for the LHeC. The different parts of the detector from the inner tracking to the outer muon chambers were presented, with the possible choices of technologies and the preliminary studies on performance. The possibilities for assembling the detector in pieces on surface to be lowered to the cavern and the estimated total time for lowering and assembly (30 months) were discussed. This is a most important point to demonstrate the feasibility of the machine that is subject to the requirement of minimal disturbance to the LHC.

On the physics at the $\mathrm{LHeC}$, Max Klein [11] revisited the perspective for precision EW measurements, providing the example of the high accuracy in the measurement of the neutral current coupling of the light quarks. On the precision QCD side and due to the huge lever arm in $Q^{2}$, the possibilities for a measurement of the strong coupling constant with a per mille accuracy, which 
demands NNNLO theoretical calculations and an accuracy in the extractions for the charm quark mass of a few $\mathrm{MeV}$, were presented.

On precision QCD studies, Max Klein [11] showed the possibilities for constraining the large and small $x$ parton densities and of performing full flavour decomposition through the combination of NC and CC. The large improvements at small $x$, and the possibilities of substantially extending the $F_{L}$ measurements done at HERA were presented.

Concerning small- $x$ physics, Néstor Armesto [9] showed the huge possibilities of the LHeC, due to its large lever arm in $x$ and to the possibilities of performing both $e \mathrm{p}$ and $e$ A collisions, for disentangling the dynamics of QCD at large energies. Using the complementarity of $e$ p and $e \mathrm{~A}$ and considering the fact that the proposed non-linear effects are density effects, the LHeC should be able to answer unambiguously the question about the presence or not of a novel non-linear regime of QCD. For that, several observables were selected with emphasis in the determination of the small- $x$ glue, the tension between different observables in standard extractions of parton densities if non-linear effects are present, and the exclusive production of vector mesons.

Hannu Paukkunen [13] stressed the huge improvements on our knowledge of parton densities inside nuclei that can be expected from the $\mathrm{LHeC}$. The machine would extend the presently explored DIS region by almost four orders of magnitude towards small $x$ and large $Q^{2}$. As an example, he showed how the present uncertainty in standard extractions of nuclear parton densities for the sea quarks and gluons at small $x$ would diminish by a large factor, a fact of uttermost importance for the studies of high-energy nuclear collisions and the search of the Quark-Gluon Plasma.

Turning to Higgs and BSM physics, Max Klein [11] and Bruce Mellado [12] discussed the impact of a precise determination of parton densities at moderate to large $x$ on the NNLO Higgs cross section in pp, with an expected accuracy of $0.25 \%$ and sensitivity to mass well below 1 $\mathrm{GeV} / \mathrm{c}^{2}$. The uncertainty in the cross section for gluino production in the gg channel was seen to reduce dramatically even for gluino masses around $5 \mathrm{TeV}$, were present uncertainties are very large. Turning to more specific Higgs physics, Bruce Mellado [12] showed the unique capabilities of the LHeC to measure the HWW vertex separately from the HZZ one, and thus to investigate physics beyond the $\mathrm{SM}$ in the $0^{+}$hypothesis with high precision.

The consequences for the yields of Higgs particles, of the cross section at the $\mathrm{LHeC}$ with integrated luminosities of order $1 \mathrm{ab}^{-1}$ achievable with instantaneous around $10^{34} \mathrm{~cm}^{-2} \mathrm{~s}^{-1}$, were discussed by Max Klein [11]. As prominent examples, 100000 Higgs into $b \bar{b}$ and 25000 into WW would be produced. In the $b \bar{b}$ channel, an expected efficiency for reconstruction around $5 \%$ is expected with $\mathrm{S} / \mathrm{N} \sim 1$, as discussed in detail by Bruce Mellado, leading to a $1 \% \mathrm{Hbb}$ coupling determination with the $\mathrm{LHeC} \mathrm{[12].}$

Effects of extensions of the SM on massive vector boson photoproduction were discussed by Magno Machado [15].

Finally, the plans for the future were discussed by Oliver Brüning [10] and Max Klein [11]. The project is entering a phase of R\&D, with CERN mandate to continue the studies towards a technical design in three years, including the development of an ERL test machine and of the ERL LHeC design. The physics case has to be further motivated specially in light of the possibilities at the LHC.

As a final summary, the LHeC is an upgrade to the LHC, complementary to the (HL-)LHC program, with implications on SM/BSM, QGP,... It has a very interesting program on its own con- 
cerning QCD in ep/A, EW physics, etc. High luminosities around $10^{34} \mathrm{~cm}^{-2} \mathrm{~s}^{-1}$ would transform the $\mathrm{LHeC}$ into a precision Higgs machine. Installation during LS3 is challenging but not impossible if resources (support) for R\&D and design are allocated soon. Steps towards a technical design in three years require refining the physics case with its relation to the LHC, developing an ERL test facility and magnets, and designing the detector.

\section{Collider experiments at the LHC, RHIC and SPS}

\subsection{Upgrade of the LHC experiments}

An excellent performance of the LHC machine and the experiments during the LHC startup stage in 2010-2012 at the centre-of-mass energy of 7 and $8 \mathrm{TeV}$ has been reported in this workshop by several speakers. The ongoing long shutdown LS1, which will extend into 2014, has the objectives to repair and consolidate the inter-magnet copper-stabilizers and the repair or replacement of several magnets. In addition, PS and SPS consolidation and upgrade work will be carried out. The goal is to allow for safe operation at design energy of $\sqrt{s}=13 \sim 14 \mathrm{GeV}$ and rich a nominal luminosity of $L \sim 10^{34} \mathrm{~cm}^{-2} \mathrm{~s}^{-1}$ with $25 \mathrm{~ns}$ bunch spacing. After the next long shutdown periods, planned for 2017-2019 (LS2) and 2022-2024 (LS3) the LHC will be upgraded for the high luminosity stage (HL-LHC), delivering of order five times the LHC nominal instantaneous luminosity $\left(5 \times 10^{34} \mathrm{~cm}^{-2} \mathrm{~s}^{-1}\right)$ along with luminosity levelling. The final goal is to extend the data set to 3000 $f b^{-1}$ by around 2030 .

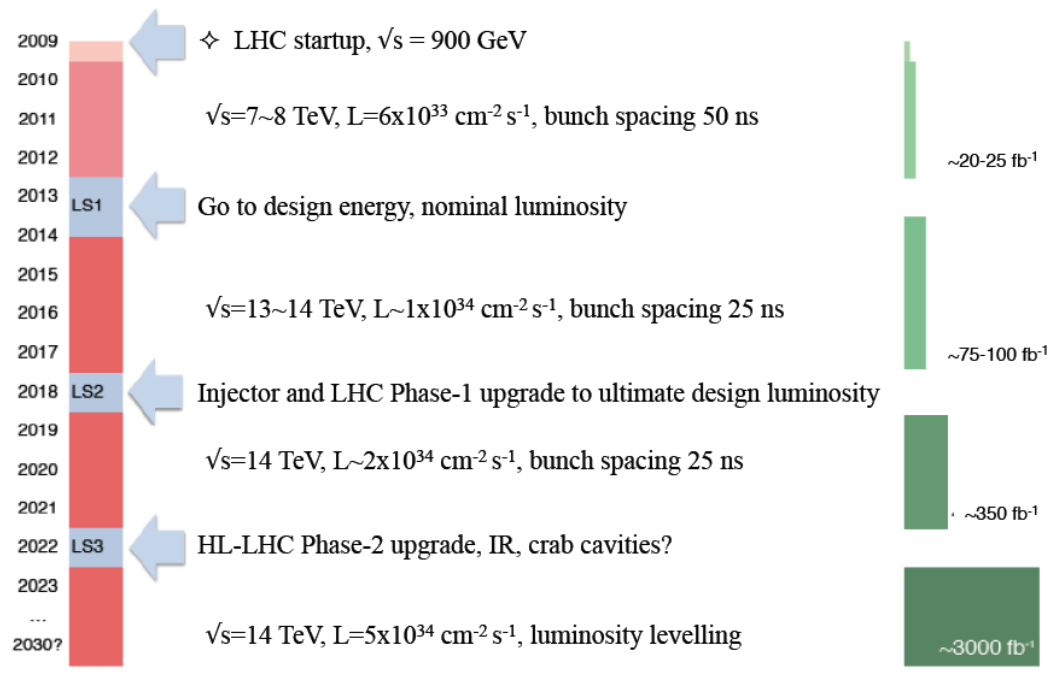

All LHC experiments will use these shutdowns to implement programme of staged modifications, consolidation, improvements and major changes to cope with high luminosity and the associated radiation level and to exploit the physics potential of the LHC. In this workshop the upgrade programs from ATLAS, ALICE and LHCb experiments have been reported [19, 20, 21].

- The upgrade programme of ATLAS experiment was presented by Silvia Miglioranzi [19]. ATLAS experiment is actively pursuing a series of upgrades to ensure continued detector efficiency and consequently optimal physics acceptance with increasing luminosity: 
- additional Pixel layer and other detector consolidation during shutdown LS1;

- major upgrades to improve Trigger capabilities during Phase-I shutdown LS2;

- replacement of the Inner Tracker, Forward Calorimeter, Electronics and Trigger/DAQ during the Phase-II shutdown LS3.

- ALICE experiment, collecting data in $P b P b, p P b$ and $p p$ collisions, aims for the characterisation of the QCD matter at high temperature and energy density. While the first running period until 2017 can provide detailed description of the global and bulk phenomena, many important measurements of rare processes, which require high statistics and high precision, particularly at low $p_{T}$, cannot be addressed in details with the current experimental setup. To meet the physics requirements of reasonable statistical and systematic uncertainties in rare probe measurements, ALICE has defined a goal to collect after LS2 about 100 times the data collected before LS2. It becomes essential to take data with a minimum bias trigger. This requires significant increase of rate capabilities for minimum bias heavy-ion collisions by recording all collision events with a fully pipe-lined read-out architecture. To achieve this goal, a major upgrades of ALICE components are foreseen, as presented in the workshop by Levente Molnar [20]. ALICE plans a major upgrade of its sub-detectors, such as Time Projection Chamber (TPC), Inner Tracking System (ITS), the read-out electronics and the data acquisition system.

- The LHCb experiment is designed to perform high-precision measurements of CP violation and search for New Physics using the enormous flux of beauty and charmed hadrons produced at the LHC. Although the LHCb detector was performing very well, the limit of $1 \mathrm{fb}^{-1}$ of data per year cannot be overcome without improving the detector. Therefore LHCb experiment plans to upgrade spectrometer by 2018 with a $40 \mathrm{MHz}$ readout and a much more flexible software-based triggering system that will increase the data rate as well as the efficiency specially in the hadronic channels. The LHCb upgrade program involves readout electronics, high level trigger and network, VELO, Calorimeters, downstream tracking, Muon systems and RICH. The detailed upgrade program was presented by Umberto Marconi [21]. The LHCb detector upgrade plans are presented in the Letter of Intent and Framework Technical Design Report.

\subsection{Upgrade of PHENIX at RHIC}

The PHENIX collaboration has developed and submitted to DOE a proposal for a greatly upgraded PHENIX detector, called sPHENIX for enhanced physics programs using full luminosity of recent upgraded RHIC facility. The project, presented in the workshop by Yuji Goto [22], provides a variety of programs with heavy-ion collisions and polarised proton collisions at RHIC. SPHENIX enables a compelling physics program that will address fundamental questions about the nature of the strongly coupled Quark-Gluon Plasma (QGP). This proposal includes a major upgrade of the central-rapidity spectrometer consisting of a solenoidal magnet surrounded by electromagnetic and hadronic calorimeters with uniform coverage over $|\eta|<1$. The upgrade enables a crucial jet physics program to investigate the nature of the strongly-coupled QGP. Additional tracking layers 
and a preshower will expand the sPHENIX program to include heavy-flavor, neutral pion, lowmass dilepton, and direct photon measurements. A new forward-rapidity spectrometer is designed for the study of cold nuclear matter effects in proton- and deuteron-nucleus collisions, precision measurements of single transverse-spin asymmetries for the Drell-Yan process, and measurements of novel observables in jet production in transversely polarised proton collisions.

\subsection{Another idea for an $e p(e A)$ collider}

Future experimental facility for the DIS experimental programme has to fulfil several conditions: it must address new physics questions, its role must be widely recognised as essential for the model independent interpretation of the LHC experimental results and as imperative for the LHC precision measurement programme, it must attract both the high energy physics and the nuclear physics communities and, last but not least, it must be cheep. Mieczyslaw Krasny has discussed the possibilities for a new electron-proton and electron-nucleus collider using the existing SPS beams and the polarised electron beam in the range of 5 to $20 \mathrm{GeV}$ from a newly built energy recovery Linac [23].

\section{Fixed Target Experiments}

\subsection{Upgrade of COMPASS-II experiment at SPS}

The COMPASS experiment at CERN has been taking data since 2002 using either muon or hadron beams with a longitudinally or transversely polarised solid target, liquid hydrogen or heavy nuclear targets. Recently the COMPASS collaboration has submitted a proposal for additional measurements in the next years, which was approved by the CERN Research Board in December 2010. The physics programs for the upgraded COMPASS-II have been discussed in the presentations of Etienne Burtin and Michela Chiosso [24, 25]. COMPASS-II experiment will study Transverse Momentum Dependent Parton Distribution Functions (TMDs) from single-polarised Drell-Yan processes using $\pi$ beam, allowing for the first time to test the sign change prediction (planned for 2014). A major part of the future COMPASS program will be dedicated to the investigation of the nucleon structure (GPDs) through Deeply Virtual Compton Scattering (DVCS) and Deeply Virtual Meson Production (DVMP) using high intensity $160 \mathrm{GeV} \mu$ beams (planned for 2015-2016). In parallel, unpolarised SIDIS measurements will be performed. The proposal includes major modifications of COMPASS spectrometer. The COMPASS is upgraded with a $4 \mathrm{~m}$-long recoil proton detector, a $2.5 \mathrm{~m}$-long liquid hydrogen and a large angle calorimeter.

\subsection{Physics with the $12 \mathrm{GeV}$ Upgrade at Jefferson Lab}

Construction of the $12 \mathrm{GeV}$ upgrade to the Continuous Electron Beam Accelerator (CEBAF) Facility at Jefferson Lab is presently underway. This upgrade program, presented in details by Cynthia Keppel [26], includes doubling the energy of the electron beam to $12 \mathrm{GeV}$, the addition of a new fourth experimental hall, and the construction of upgraded detector hardware. The result will be an exciting physics program, providing new insights into the structure of the nucleon, the transition between the hadronic and quark-gluon descriptions of matter, and the nature of quark confinement. The approved experimental program includes more than 50 measurements to be 
conducted within the next 7 years. The operation of experiments is expected to resume in February 2014.

\subsection{AFTER@LHC: A Fixed-Target ExpeRiment at the LHC}

The physics opportunities offered by a proposed multi-purpose fixed-target experiment exploiting the LHC beams extracted by a bent crystal (AFTER@LHC) have been discussed by Andry Rakoyozafindrabe [27]. The beam extraction using a strong crystalline field technique offers a clean and very collimated high-energy beam, without altering the LHC performance. The feasibility of this technique has been already studied with SPS beam. A LUA9 proposal to perform these tests with the LHC beam has been approved by the LHCC.

The very energetic LHC beams will allow the measurements of $p p, p d$ and $p A$ collisions at $\sqrt{s_{N N}} \sim 115 \mathrm{GeV}$ and $\mathrm{PbA}$ collisions at $\sqrt{s_{N N}} \sim 72 \mathrm{GeV}$. A multipurpose experiment based on the modern detection techniques will give access to new domains of particle and nuclear physics complementing that of collider experiments, e.g. at RHIC. Together with a typical advantages of a fixed target experiment the instantaneous luminosity achievable with AFTER in $p p$ and $p A$ mode surpasses that of RHIC by more than 3 orders of magnitude and is comparable to that of the LHC collider mode. This provides a quarkonium and heavy-flavour observatory in $p p$ and $p A$ collisions. The instrumentation in the target region will give access to the gluon and heavy quark distributions of the proton, neutron and the nuclei at large $x$. The nuclear target-species versatility provides a unique opportunity to study nuclear matter versus the features of the hot and dense matter formed in heavy-ion collisions, including the formation of the QGP. The fixed-target mode also has the advantage to allow for spin measurements with polarised targets.

Target schedule for this project is the installation during the LHC long shutdown LS3.

\subsection{CHIC: Charm in Heavy Ion Collisions}

Since the pioneering studies of quarkonium production in heavy ion collisions at the SPS and the observation of an anomalous suppression of $J / \psi$ production in $P b P b$ collisions by NA50, these quarkonium studies have been extended to regimes of significantly higher energies at RHIC and the LHC. In addition to the results obtained at RHIC on $J / \psi$ and at the LHC on quarkonium states, hints of the theoretically expected sequential suppression start to emerge. Nevertheless, the experimental validations of such a scenario as well as the characterisation of the phase transition require full control of the feed-down sequence. In particular, a precise measurement of quarkonium $1 P$ states which significantly contribute to the yields of quarkonium $1 S$ states is mandatory. For charmonium, the measurement of $\chi_{c}$ production together with $J / \psi$ and $\psi^{\prime}$ is needed to prove the sequential suppression scenario. A new experiment at the CERN SPS is proposed, which makes use of modern ultra-granular technologies to perform the measurement of $\chi_{c} \rightarrow J / \psi+\gamma$ in $\mathrm{PbPb}$ high multiplicity collisions. The project was presented in the workshop by Frederic Fleuret [28]. The Expression of Interest presented to SPSC has been positively evaluated. Once accepted, it will take about five years for the simulation, final design, construction and commissioning.

\subsection{Neutrino-nucleus scattering with $v$ STORM}

The proposed $v$ STORM facility, presented by Ian Taylor [29], will provide $v_{e}$ and $v_{\mu}$ beams from the decay of $3.8 \mathrm{GeV}$ muons confined within a storage ring. The proposals are being prepared 
for facilities at either FNAL or CERN. A detector placed approximately $1500 \mathrm{~m}$ from the end of the decay straight, combined with a near detector, can be used for searches for sterile neutrinos. The neutrino and anti-neutrino event rates are such that the $v$ STORM facility serving a suite of near detectors will be able to measure $v_{e} N$ and $v_{\mu} N$ cross sections with the per cent-level precision required to allow the next generation of long-baseline neutrino-oscillation experiments to fulfil their potential. The status of the $v$ STORM project was presented along with the performance of the facility in terms of sterile-neutrino sensitivity and precision on measurements of neutrino-nucleus scattering.

\subsection{NA62 experiment: search for $K \rightarrow \pi v v$ at SPS}

The rare decay $K \rightarrow \pi v v$ is an excellent process to test new physics at the highest scale. The NA62 experiment at CERN SPS, described by Antonio Cassese [30], aims to collect of the order of about hundred $\mathrm{K}^{+} \rightarrow \pi^{+} v v$ events within two years of data taking, keeping the background at the level of $10 \%$. Part of the experimental apparatus has been commissioned during a technical run in 2012. The data taking will start in 2014.

\section{Data Preservation in High Energy Physics}

The international study group on data preservation in high energy physics, DPHEP, achieved a milestone in 2012 with the publication of its eagerly anticipated large scale report, which contains a description of data preservation activities from all major high-energy physics collider-based experiments and laboratories. A success of the data preservation in HEP requires long term investment in hardware and human resources. With this in mind DPHEP will evolve to a new collaboration structure in 2013. The statements from the DPHEP study group and the major conclusions from the report have been presented by Dave South [31] along with an outline of the future working directions of the new collaboration.

\section{Acknowledgements}

We wish to thank all speakers in our working group sessions and the Organising Committee for the interesting and stimulating workshop.

\section{References}

[1] D. Boer et al., Gluons and the quark sea at high energies: Distributions, polarization, tomography, arXiv:1108.1713 [nucl-th].

[2] A. Accardi et al., Electron Ion Collider: The Next QCD Frontier - Understanding the glue that binds us all, arXiv:1212.1701 [nucl-ex].

[3] A. Deshpande, these proceedings.

[4] M. Lamont, these proceedings.

[5] E-C. Aschenauer, these proceedings.

[6] K. Dehmelt, these proceedings. 
[7] Y. Zhang, these proceedings.

[8] P. Nadel-Turonski, these proceedings.

[9] N. Armesto, these proceedings.

[10] O. Brüning, these proceedings.

[11] M. Klein, these proceedings.

[12] B. Mellado, these proceedings.

[13] H. Paukkunen et al. [Group for the LHeC Study Collaboration], arXiv:1306.2486 [hep-ph].

[14] D. South, these proceedings.

[15] M. Machado, these proceedings.

[16] J. L. Abelleira Fernandez et al. [LHeC Study Group Collaboration], J. Phys. G 39 (2012) 075001 [arXiv:1206.2913 [physics.acc-ph]]; http:// cern.ch/1hec.

[17] J. L. Abelleira Fernandez et al. [LHeC Study Group Collaboration], arXiv:1211.4831 [hep-ex]; arXiv:1211.5102 [hep-ex].

[18] O. Brüning and M. Klein, Mod. Phys. Lett. A 28 (2013) 16, 1330011 [arXiv:1305.2090 [physics.acc-ph]].

[19] S. Miglioranzi, these proceedings.

[20] L. Molnar, these proceedings.

[21] U. Marconi, these proceedings.

[22] Y. Goto, these proceedings.

[23] M.W. Krasny, these proceedings.

[24] E. Burtin, these proceedings.

[25] M. Chiosso, these proceedings.

[26] C. Keppel, these proceedings.

[27] A. Rakoyozafindrabe, these proceedings.

[28] F. Fleuret, these proceedings.

[29] I. Taylor, these proceedings.

[30] A. Cassese, these proceedings.

[31] D. South, these proceedings. 